\title{
Iron, zinc, copper and magnesium nutritional status in Mexican children aged 1 to $l 1$ years
}

\author{
Ma. del Carmen Morales-Ruán, MSc, (I) Salvador Villalpando, MD, PhD, (1) \\ Armando García-Guerra, MSc,(I) Teresa Shamah-Levy, MSc, (I) Ricardo Robledo-Pérez, PhD,(I) \\ Marco Antonio Ávila-Arcos, (I) Juan A Rivera, PhD.(I)
}

\section{Morales-Ruán MC, Villalpando S, García-Guerra A, Shamah-Levy T, Robledo-Pérez R, Ávila-Arcos MA, Rivera JA. Iron, zinc, copper, and magnesium nutritional status in Mexican children aged I to II years. Salud Publica Mex 2012;54:125-134.}

\begin{abstract}
Objective. To describe the micronutrient nutritional status of a national sample of I-II year old Mexican children surveyed in 2006 in National Health and Nutrition Survey (ENSANUT 2006) and their association with dietary and sociodemographic factors. Materials and methods. Serum samples were used $(n=5060)$ to measure the concentrations of ferritin, transferrin receptor, zinc, copper and magnesium. Results. Prevalence of deficiencies in $\mathrm{I}-4$ and 5 - Ily old children were for iron (using low ferritin) 26.0 and $13.0 \%$; zinc, $28 . I$ and $25.8 \%$, respectively; and copper, $\approx 30 \%$ in both age groups. Magnesium low serum concentrations (MLSC), were found in $12.0 \%$ and $28.4 \%$ of the children, respectively. Being beneficiary of Liconsa (OR=0.32; C.I.95\%, 0.17-0.6I) or belonging to higher socioeconomic status (OR=0.63; C.I.95\%, 0.4 I-0.97) were protective against iron deficiency. Increasing age (OR=0.59; C.I.95\%, I.19-I.32) and living in the Central Region (OR=0.59; C.I.95\%, 0.36-0.97) were protective against MLSC. Conclusions. Deficiencies of iron and zinc are serious public health problems in Mexican children.
\end{abstract}

Key words: Iron; zinc; magnesium; copper; children; Mexico
Morales-Ruán MC, Villalpando S, García-Guerra A, Shamah-Levy T, Robledo-Pérez R, Ávila-Arcos MA, Rivera JA. Estado nutricio de hierro, zinc, cobre y magnesio en niños mexicanos de I a I I años de edad. Salud Publica Mex 20 I2;54:I25-I 34.

\section{Resumen}

Objetivo. Describir el estado nutricio de micronutrimentos en niños de I-I I años de edad de la Encuesta Nacional de Salud y Nutrición 2006 y su asociación con factores dietéticos y sociodemográficos. Material y métodos. Se usaron muestras séricas $(n=5060)$ para medir las concentraciones de ferritina, receptor de transferrina, zinc, cobre y magnesio. Resultados. La prevalencias de deficiencias en niños de $\mathrm{I}-4$ y de 5 a II años fueron para ferritina, 26.0 y I $3 \%$; zinc, 28 .I y $25.8 \%$ respectivamente y cobre $\approx 30 \%$ en ambos grupos. Las concentraciones bajas de magnesio (CBM) fueron 12.0 y $28.4 \%$, respectivamente. Ser beneficiario de Liconsa (RM=0.32; IC 95\%: 0.17-0.6I) y pertenecer al nivel socioeconómico alto (RM=0.63; IC, 95\%: 0.4I-0.97) fueron protectores para deficiencia de hierro. La edad (RM=I.26; IC, 95\%: I. 19-I.32) y vivir en la región Centro (RM=0.59; IC, 95\%: 0.36-0.97) fueron protectores para CBM. Conclusiones. Las deficiencias de hierro y zinc son serios problemas de salud pública en niños mexicanos.

Palabras clave: hierro; zinc; magnesio; cobre; niños; México

(I) Centro de Investigación en Nutrición y Salud, Instituto Nacional de Salud Pública. Cuernavaca, Morelos, México.

Received on: August 3I, 20II - Accepted on: February I, 2012

Corresponding author: Dr. Salvador Villalpando, PhD. Dirección de Vigilancia de la Nutrición, Centro de Investigación en Nutrición y Salud, Instituto Nacional de Salud Pública. Av. Universidad 655, Santa María Ahuacatitlán. 62100, Cuernavaca, Morelos, México.

E-mail: svillalp@insp.mx 
The prevalence of vitamin and mineral deficiencies 1 is high in developing countries. ${ }^{1}$ Children and pregnant and lactating women are the most vulnerable groups. In preschool age children, micronutrient deficiencies increase the risk for acute diarrhea and pneumonia. These deficiencies are responsible for a great number of deaths and a large proportion of the disease burden worldwide ${ }^{2}$ and also impinges on economic and human capital development. ${ }^{3}$

Iron deficiency impairs immune function and limits cognitive development in children, causes low productivity in adults and increases the risk of perinatal death. ${ }^{4,5}$ Zinc deficiency affects children's physical growth and increases the risk and severity of diarrhea, pneumonia and other infections. ${ }^{6,7}$ Magnesium is a cofactor of more than 300 enzymes; participates in the synthesis of proteins and nucleic acids, neuromuscular transmission, and in cardiac contraction. ${ }^{8}$ Magnesium deficiency is linked to malabsorption and in children has been associated with insulin resistance. ${ }^{8-10}$ Copper participates in the synthesis of connective tissue and the myelin sheath of nerves, as well as in energy and iron metabolism, is the reductive agent of iron oxidase enzymes and is a constituent of the ceruloplasmins involved in iron transport and absorption. ${ }^{11}$ Copper deficiency manifests as hypochromic anemia, and in children has been associated with osteoporosis and stunting. ${ }^{12,13}$

The Mexican National Health and Nutrition Survey 1999 (ENN 99) reported a high prevalence of iron (41.4\%) and zinc $(25.3 \%)$ deficiencies in children younger than 11 years. ${ }^{14}$ The aim of this study is to describe the prevalence and distribution of iron and zinc deficiency and low serum concentration (LSC) of copper and magnesium in a probabilistic sample of Mexican children aged 1 to 11 years participating in the National Health and Nutrition Survey 2006 (ENSANUT 2006).

\section{Materials and methods}

Data for this analysis were collected from ENSANUT 2006 database. The survey had a probabilistic, clustered sample design and was representative at national, urban, rural and regional (Northern, Center, Southern, and Mexico City) level. The methodology and calculation of the sample size have been published elsewhere. ${ }^{15}$

Study sample. Data from children aged 1 to 11 years from the ENSANUT 2006 corresponding to $30 \%$ of the overall sample were extracted.

\section{Data collection}

Socioeconomic status (SES). SES data were obtained using a validated questionnaire including age, sex, years of schooling, and being beneficiary of two Federal social programs with nutrition components (Oportunidades or Liconsa). A SES indicator was constructed using a Principal Component Analysis, based on characteristics and assets of households. The validation of the method has been published elsewhere. ${ }^{16}$

Dietary intake. Dietary information was collected using a semiquantitative food frequency questionnaire including 101 foods classified into 14 groups. For each food, number of days of intake per week, times a day, portion size (very small, small, medium, large, and very large), mean weight of portion and number of portions consumed within the seven days before the date of interview were asked. ${ }^{17}$ Total energy and nutrients contained in each food consumed and in total diet for each individual were estimated through the Nutrient Composition Database compiled by the INSP.

Micronutrient nutritional status. A venous blood sample was drawn into mineral-free Vacutainer evacuated tubes (Beckton Dickinson Inc). Thirty minutes prior the centrifugation process, the samples were kept inside an ice container. The blood was spun-down at $2500 \mathrm{~g}$, in situ, serum was separated and aliquoted into cryovials to be transported to the Nutrition Laboratory of the INSP placed in liquid nitrogen. All material used for related procedures was acid-washed or mineral-free.

Laboratory determinations. The serum concentrations of ferritin, and soluble transferrin receptors (sTfR) were measured by immunoassay method using commercial kits (Dade Behring Inc.). C reactive protein (CRP) was measured by nephelometry, using ultrasensitive monoclonal antibodies (Behring Nephelometer 100 Analyzer). Coefficients of variation were: ferritin $4.71 \%$, sTfR $5.6 \%$ and CRP $4.20 \%$.

Serum concentrations of copper, zinc, and magnesium were determined by inductively coupled plasma optical emission spectrometry Varian Vista Pro CCD Simultaneous. The results were calibrated against NIST SRM 3131a reference material. Coefficients of variation were: copper, $2.1 \%$, zinc $2.4 \%$ and magnesium $11.8 \%$.

Iron deficiency was assessed using two separate indicators: Low iron stores, ferritin $<12 \mu \mathrm{g} / \mathrm{L}$ (LIS) ${ }^{18}$ and tissue iron deficiency (TID), sTfR $>6 \mathrm{mg} / \mathrm{L}^{19}$

Zinc deficiency was defined according to the time of the day and fasting hours at the moment of the sample collection. In children (girls and boys) under nine years old values under $65 \mu \mathrm{g} / \mathrm{dL}$ and $57 \mu \mathrm{g} / \mathrm{dL}$ were classified 
as deficient. In the age group of 9 to 11 years deficiency was defined as follows: values under $70 \mu \mathrm{g} / \mathrm{dL}$ for boys and $74 \mu \mathrm{g} / \mathrm{dL}$ for girls in the case of samples taken fasting in the morning. ${ }^{20}$

Magnesium LSC was defined as serum concentrations $<0.75 \mathrm{mmol} / \mathrm{L}^{9,21}$ and copper LSC, if concentrations $<90 \mu \mathrm{g} / \mathrm{dL}^{22}$ High CRP values were considered abnormal when serum concentration was $>6 \mathrm{mg} / \mathrm{L}$.

\section{Statistical analysis}

Descriptive statistics included central tendency and dispersion indicators. Logistic regression models were constructed to test the association between deficiency or LSC of minerals and sociodemographic and dietary variables, adjusting for age, sex, SES, being beneficiary of Oportunidades or Liconsa programs and dietary intake. The zinc, iron (using ferritin), copper and magnesium models were adjusted for CRP to control for effect of inflammation.

Estimators were calculated for each micronutrient (except sTfR) including and excluding the children with high CRP in order to test if differences exists between such populations.

\section{Association with $p<0.05$ were considered significant}

Because of oversampling of the poorest population from the Southern Region, a procedure based on regression models estimates was used to reduce possible selection bias in the selected samples. Population means for height, weight, hemoglobin and the estimated SES index for region, age, sex, area, and strata were included in the estimators. The original expansion factors of the survey were recalculated to improve the population representativeness for estimators at the national level. Analyses were performed using Stata software (Version 10.1) SVY module for complex samples and SPSS software (Version 16.0).

Ethical aspects. The parents or guardians of the children signed an informed consent letter after a careful explanation of the objectives, risks and benefits of the survey.

The protocol was approved by the Research, Ethics and Biosecurity, Committees of the National Institute of Public Health, Cuernavaca, Mexico.

\section{Results}

Data from 5060 children aged 1 to 11 years, representing 24 million children nationwide, were analyzed. Distribution was not different by gender (boys 50.1\%), or age strata. Urban children were $72.5 \%$, low SES 40\%,
The Southern region was overrepresented with $33.9 \%$ of the sample; potential bias was corrected by recalculating the expansion factors (Table I).

The percentage of children with $C$ reactive protein $(\mathrm{CRP})>6 \mathrm{ml} / \mathrm{L}$ was determined. Children of 1 to 2 years old showed a prevalence of $9.9 \%$ (95\% CI: 7.4-13.2), the group of 3 to 4 years old $9.1 \%$ (95\% CI:6.9 -11.9), 5 to 6 $8.4 \%$ (95\% CI: $6.5-11.0)$, 7 to 8 of $7.9 \%$ (95\% CI: $6.0-10.5$ ), 9 to 10 of $7.4 \%$ (95\% CI: $5.3-10.2)$ and finally in the 11 years old group the prevalence was $6.9 \%$ (95\% CI: 4.9-9.6). An overall percentage of $8.4 \%$ (95\% CI: 6.4-11.2) for high CRP for all age groups was obtained.

\section{Nutritional status of iron}

The median of serum ferritin concentrations was 28.7 $\mu \mathrm{g} / \mathrm{L}$ (interquartile range [IQR]: $16.5,45.4 \mu \mathrm{g} / \mathrm{L}$ ). The

Table I

\section{Characteristics of the population}

\begin{tabular}{lccc} 
& $n$ & $\%$ & $N$ (thousands) \\
$\begin{array}{c}\text { Gender } \\
\text { Boys }\end{array}$ & 2582 & 50.1 & II 886.148 \\
\hline Girls & 2478 & 49.9 & II 841.662
\end{tabular}

Age group (years)

\begin{tabular}{lrrr}
$1-2$ & 665 & 15.1 & 3571.0 \\
\hline $3-4$ & 1140 & 19.4 & 4594.0 \\
\hline $5-6$ & 1582 & 18.7 & 4439.2 \\
\hline $7-8$ & 620 & 17.7 & 4199.5 \\
\hline $9-10$ & 704 & 20.7 & 4908.9 \\
\hline 11 & 349 & 8.5 & 2015.2
\end{tabular}

Area

\begin{tabular}{crrr} 
Urban & 2842 & 72.5 & 17191.9 \\
\hline Rural & 2218 & 27.5 & 6535.9
\end{tabular}

\begin{tabular}{lrrr}
$\begin{array}{l}\text { Region } \\
\text { Northern }\end{array}$ & 795 & 19.0 & 4518.0 \\
\hline Center & 1911 & 31.8 & 7554.2 \\
\hline Mexico City & 238 & 15.2 & 3601.4 \\
\hline Southern & 2116 & 33.9 & 8054.2
\end{tabular}

Socioeconomic status*

\begin{tabular}{|c|c|c|c|}
\hline Low & 2664 & 40.6 & 9596.4 \\
\hline Middle & 1635 & 33.6 & 7949.4 \\
\hline High & 751 & 25.8 & 6106.5 \\
\hline \multicolumn{4}{|c|}{ Beneficiary of food assistance programs } \\
\hline Oportunidades & 2981 & 59.0 & 14006.5 \\
\hline Liconsa & 589 & 11.7 & 2766.7 \\
\hline
\end{tabular}

* Score obtained by principal components according to household characteristics 
overall prevalence of LIS (ferritin $<12 \mu \mathrm{g} / \mathrm{L}$ ) was $17.6 \%$ (95\% CI: 14.2-21.8). The highest prevalence occurred in children 1- 2 years old $(31.7 \%, 95 \%$ CI: 26.2-37.8) and decreased as age increased. The prevalence in children younger than 5 years was 26.0\% (95\% CI: 21.3-31.3) and in 5-11 years old children, $13.0 \%$ (95\% CI: 10.2-16.5) (Table II). No significant differences were found between rural and urban children (19.6\%, 95\% CI: 15.9-24.0 and 16.1\%, 95\% CI: 12.8-20.0). The Northern and Southern regions showed the highest prevalence $(\approx 20 \%)$ and Mexico City, the lowest (4.5\%; 95\% CI: 2.3-8.6) (Table III).

In a logistic regression model, being beneficiary of the fortified milk Liconsa, (OR=0.32; 95\% CI: 0.17, $0.61)$, and high SES (RM=0.63; CI, 95\%: $0.41,0.97)$ were protective for the risk of LIS (ferritin $<12 \mathrm{ug} / \mathrm{L}$ ).

The median sTfR concentration was $4.4 \mathrm{mg} / \mathrm{L}$ (IQR: 3.7, $5.1 \mathrm{mg} / \mathrm{L}$ ). The overall prevalence of TID (sTfR $>6 \mathrm{mg} / \mathrm{L}$ ) was 10.7\% (95\% CI: 8.1-14.0); the highest in children 1-2 years old (18.6\%; 95\% CI: 14.2-24.1) and decreased as age progressed. The prevalence was higher in children younger than 5 years $(15.7 \%$; $95 \%$ CI: 12.0-20.3) compared with 5-11 years old children (7.9\%; 95\% CI: 5.9-10.6) (Table II). No differences between urban and rural children or among regions were noted (Table III).

In a logistic regression model living in Mexico City represented a risk of TID (OR=1.96; 95\% CI: 1.06-3.63) and increasing age was protective $(\mathrm{OR}=0.92 ; 95 \% \mathrm{CI}$ : 0.85-0.99) for the risk for TID.

No association was found between TID and the intakes of total iron or iron absorption inhibitors, region, area of residence, SES and being beneficiary of Liconsa or Oportunidades. (Table IV).

\section{Nutritional status of zinc}

The overall, mean serum zinc concentration was 94.9 $\mu \mathrm{g} / \mathrm{dL}$ (95\% CI: 89.5-100.3 $\mu \mathrm{g} / \mathrm{dL})$. The overall prevalence of zinc deficiency was $26.6 \%$ (95\% CI: 21.4-32.6). No significant differences were found among age groups or between rural and urban areas (Tables II and III). Prevalence was higher in the Northern region (32.4\%; 95\% CI: 26.0-39.4) with no significant differences among other regions. No significant association was found among risk for zinc deficiency and dietary and sociodemographic variables (data not shown).

\section{Nutritional status of copper}

The overall mean serum copper concentration was 105.2 $\mu \mathrm{g} / \mathrm{dL}$ (95\% CI: 101.8, 108.6 $\mu \mathrm{g} / \mathrm{dL}$ ) (Table II). Overall copper LSC (<90 $\mu \mathrm{g} / \mathrm{dL}$ ) was 30.6\% (95\% CI: 25.2-36.7). No significant differences were observed by age group, area of residence, or geographic region (Table III). In a logistic regression model, living in the Southern region $(\mathrm{OR}=0.64 ; 95 \%$ CI: $0.41-0.99)$ and being male $(\mathrm{OR}=0.76$; 95\% CI: 0.62-0.94) were protective and being beneficiary of Liconsa (OR=1.92; 95\% CI: 1.24-2.97) or Oportunidades $(\mathrm{OR}=1.49 ; 95 \%$ CI: 1.02-2.16) programs represented a risk (Table IV).

\section{Nutritional status of magnesium}

The overall mean serum magnesium concentration was $0.86 \mathrm{mmol} / \mathrm{L}$ (95\% CI: $0.83-0.88 \mathrm{mmol} / \mathrm{L}$ ). The overall proportion of children with magnesium LSC $(<0.75$ $\mathrm{mmol} / \mathrm{L}$ ) was $22.6 \%$ (95\% CI: 17.5-28.6). The lowest proportion occurred in children 1-2 years old $(9.1 \%$; 95\% CI: 6.6-12.5) and increased with progressing age up to $46.6 \%$ (95\% CI: 37.3-56.2) in 11-year-old children (Table II). The proportion of magnesium LSC in children younger than 5 years was $12.0 \%$ (95\% CI: 9.0-16.0); that is, 16 percentage points lower than children $5-11$ years old (28.4\%; 95\% CI: 22.2-35.5). The proportion was higher in urban $(25.8 \%, 95 \%$ CI: 20.1-32.3) than in rural (18.6\%, 95\% CI: 14.2-24.0). The proportion of LSC was significantly higher in Mexico City (30.6\%, 95\% CI: 19.244.67), and lowest in the Center region (18.6\%, 95\% CI: 14.1-23.9) (Table III). In a logistic regression model, living in the Center region was protective $(\mathrm{OR}=0.57 ; 95 \% \mathrm{CI}$ : $0.35-0.93)$ while age represented a risk $(\mathrm{OR}=2.34 ; 95 \%$ CI: 1.75-3.12).

\section{Discussion}

We found that iron and zinc deficiency, particularly in children 1 to 2-year-old remain as public health problems in Mexico. Likewise, low serum concentrations of magnesium and copper LSC are high in Mexican children, although the health implications of these low serum values are not known.

\section{Nutritional status of iron}

The greater risk for iron deficiency observed in 1-2-yearold children may be explained by the early depletion of body iron stores and because of the high demand of iron to expand the erythrocytic mass during that period of life. Such demand cannot be met by the usual iron intake during the weaning period. 23,24

The prevalence of iron deficiency decreased from $41.4 \%$ in $1999^{14}$ to $12.0 \%$ in 2006 in 5-11-year-old children. However, the prevalences are not comparable between surveys since different indicators were used for assessing iron deficiency, total iron binding capacity (TIBC) in 1999 and ferritin concentrations in 2006. 
Table II

Serum Concentrations of Minerals AND PREVALENCE OF DEFICIENCY (OR LOW SERUM CONCENTRATIONS) in Children I to I I years old, by age group. National Health and Nutrition Survey 2006

\begin{tabular}{|c|c|c|c|c|c|c|c|c|c|}
\hline \multirow[b]{2}{*}{ Age group } & \multicolumn{3}{|c|}{ Ferritin $(\mu \mathrm{g} / \mathrm{L})$ centile } & \multicolumn{4}{|c|}{ Prevalence of low iron stores, (ferritin $<12.0 \mu \mathrm{g} / \mathrm{L}$ ) } & \multicolumn{2}{|c|}{ Total population } \\
\hline & 25 & 50 & 75 & $N$ (thousands) & $n$ & $\%$ & $\mathrm{Cl}(95 \%)$ & $N$ (thousands) & $n$ \\
\hline $1-2$ & 8.2 & 18.3 & 30.2 & I 132.0 & 219 & 31.7 & $(26.2,37.8)$ & 3571.0 & 625 \\
\hline $3-4$ & 14.7 & 25.2 & 40.5 & I 047.4 & 213 & 22.8 & $(18.6,27.7)$ & 4594.0 & 1085 \\
\hline $5-6$ & 16.4 & 27.6 & 41.5 & 736.9 & 262 & 16.6 & $(13.4,20.6)$ & 4439.2 & 1557 \\
\hline $7-8$ & 21.8 & 34.1 & 52.3 & 487.1 & 49 & 11.6 & $(9.1,14.8)$ & 4199.5 & 616 \\
\hline $9-10$ & 23.9 & 40.0 & 59.6 & 427.1 & 72 & 8.7 & $(6.5, I I .7)$ & 4908.9 & 689 \\
\hline II & 21.2 & 40.9 & 61.1 & 143.1 & 33 & 7.1 & $(5.2,9.8)$ & 2015.2 & 344 \\
\hline$<5$ & 12.9 & 22.7 & 36.1 & 2122.9 & 432 & 26.0 & $(21.3,31.3)$ & 8165.1 & 1710 \\
\hline $5-11$ & 19.2 & 32.2 & 49.1 & 2023.2 & 416 & 13.0 & $(10.2,16.5)$ & 15562.7 & 3206 \\
\hline Total & 16.5 & 28.7 & 45.4 & 4 I76.I & 848 & 17.6 & $(|4.2,2| .8)$ & 23727.8 & 4916 \\
\hline
\end{tabular}

\begin{tabular}{|c|c|c|c|c|c|c|c|c|c|}
\hline \multirow[b]{2}{*}{$\mathrm{I}-2$} & \multicolumn{3}{|c|}{$s T f R(m g / L)$} & \multicolumn{4}{|c|}{ Prevalence of tissue iron deficiency $(s T f R>6 \mathrm{mg} / \mathrm{L})$} & \multirow[b]{2}{*}{3571.0} & \multirow[b]{2}{*}{626} \\
\hline & 4.1 & 4.8 & 5.7 & 664.2 & 137 & 18.6 & $(14.2,24.1)$ & & \\
\hline $3-4$ & 3.8 & 4.5 & 5.1 & 643.2 & 134 & 14.0 & $(10.8,18.1)$ & 4594.0 & 1081 \\
\hline $5-6$ & 3.6 & 4.3 & 5.1 & 443.9 & 150 & 10.0 & $(7.7,13.1)$ & 4439.2 & 1557 \\
\hline $7-8$ & 3.8 & 4.4 & 5.2 & 310.8 & 46 & 7.4 & $(5.5,9.9)$ & 4199.5 & 614 \\
\hline $9-10$ & 3.7 & 4.3 & 5.0 & 260.2 & 46 & 5.3 & $(3.7,7.6)$ & 4908.9 & 689 \\
\hline II & 3.5 & 4.3 & 5.1 & 78.6 & 18 & 3.9 & $(2.7,5.8)$ & 2015.2 & 341 \\
\hline$<5$ & 3.9 & 4.6 & 5.3 & 1281.9 & 271 & 15.7 & $(12.0,20.3)$ & 8165.1 & 1707 \\
\hline $5-11$ & 3.6 & 4.3 & 5.1 & I 229.5 & 260 & 7.9 & $(5.9,10.6)$ & 15562.7 & 3201 \\
\hline Total & 3.7 & 4.4 & 5.1 & 2538.9 & 531 & 10.7 & $(8.1,14.0)$ & 23727.8 & 4908 \\
\hline
\end{tabular}

\begin{tabular}{|c|c|c|c|c|c|c|c|c|}
\hline & \multicolumn{2}{|c|}{$\operatorname{Zinc}(\mu g / d L)$} & \multicolumn{4}{|c|}{ Prevalence of zinc deficiency* } & & \\
\hline & Mean & $\mathrm{Cl}(95 \%)$ & & & & & & \\
\hline $\mid-2$ & 92.1 & $(86.5,97.8)$ & I 042.8 & 164 & 29.2 & $(23.8,35.3)$ & 3571.0 & 562 \\
\hline $3-4$ & 93.4 & $(88.2,98.6)$ & I 264.4 & 272 & 27.5 & $(22.5,33.2)$ & 4594.0 & 988 \\
\hline $5-6$ & 94.9 & $(89.7,100.0)$ & I 173.7 & 375 & 26.4 & $(21.5,32.1)$ & 4439.2 & 1419 \\
\hline $7-8$ & 96.0 & $(90.7,101.4)$ & I 076.2 & 121 & 25.6 & $(20.6,31.5)$ & 4199.5 & 472 \\
\hline $9-10$ & 97.3 & $(91.3,103.3)$ & I 224.3 & 133 & 24.9 & $(19.4,31.6)$ & 4908.9 & 534 \\
\hline II & 98.6 & $(92.3,104.8)$ & 493.8 & 63 & 24.5 & $(|8.8,3| .4)$ & 2015.2 & 259 \\
\hline$<5$ & 92.9 & $(87.6,98.3)$ & 2296.8 & 436 & 28.1 & $(23.0,34.0)$ & 8165.1 & 1550 \\
\hline $5-11$ & 96.0 & $(90.5,101.5)$ & 4009.8 & 692 & 25.8 & $(20.6,31.8)$ & 15562.7 & 2684 \\
\hline Total & 94.9 & $(89.5,100.3)$ & 6613.1 & 1127 & 26.6 & $(21.4,32.6)$ & 23727.8 & 4234 \\
\hline
\end{tabular}

\begin{tabular}{|c|c|c|c|c|c|c|c|c|}
\hline & \multicolumn{2}{|c|}{ Copper ( $\mu \mathrm{g} / \mathrm{dL})$} & \multicolumn{4}{|c|}{ Prevalence of low serum copper concentration $(<90 \mu \mathrm{g} / \mathrm{dL})$} & \multirow[b]{3}{*}{3571.0} & \multirow[b]{3}{*}{468} \\
\hline & Mean & $\mathrm{Cl}(95 \%)$ & & & & & & \\
\hline $1-2$ & 104.8 & $(101.3,108.3)$ & I 063.8 & 113 & 29.8 & $(24.4,35.9)$ & & \\
\hline $3-4$ & 104.3 & $(I 0 I . I, I 07.5)$ & | 419.5 & 230 & 30.9 & $(25.9,36.4)$ & 4594.0 & 808 \\
\hline $5-6$ & 104.7 & $(101.5,107.8)$ & I 387.4 & 562 & 31.3 & $(26.2,36.8)$ & 4439.2 & $|49|$ \\
\hline $7-8$ & 105.7 & $(102.3,109.1)$ & I 296.2 & 135 & 30.9 & $(25.3,37.1)$ & 4199.5 & 494 \\
\hline $9-10$ & 106.9 & $(102.9,110.9)$ & I 470.7 & 139 & 30.0 & $(23.7,37.5)$ & 4908.9 & 589 \\
\hline II & 107.6 & $(103.5,111.8)$ & 594.5 & 92 & 29.5 & $(23.0,37.0)$ & 2015.2 & 285 \\
\hline$<5$ & 104.5 & $(101.2,107.8)$ & 2490.2 & 343 & 30.5 & $(25.3,36.2)$ & 8165.1 & 1276 \\
\hline $5-11$ & 105.6 & $(102.2,109.1)$ & $478 \mid .4$ & 928 & 30.7 & $(25.2,37.0)$ & 15562.7 & 2859 \\
\hline Total & 105.2 & $(101.8,108.6)$ & 7260.7 & $|27|$ & 30.6 & $(25.2,36.7)$ & 23727.8 & 4135 \\
\hline
\end{tabular}

\begin{tabular}{|c|c|c|c|c|c|c|c|c|}
\hline & \multicolumn{2}{|c|}{ Magnesium (mmol/L) } & \multicolumn{4}{|c|}{ Low serum magnesium concentrations ( $<0.75 \mathrm{mmol} / \mathrm{L})$} & & \\
\hline & Mean & $\mathrm{Cl}(95 \%)$ & & & & & & \\
\hline I-2 & 0.89 & $(0.87,0.91)$ & 324.6 & 72 & 9.1 & $(6.6,12.5)$ & 3571.0 & 467 \\
\hline $3-4$ & 0.88 & $(0.85,0.90)$ & 629.8 & 107 & 13.7 & $(10.3,18.1)$ & 4594.0 & 807 \\
\hline $5-6$ & 0.86 & $(0.84,0.88)$ & 891.2 & 234 & 20.1 & $(15.5,25.6)$ & 4439.2 & 1489 \\
\hline $7-8$ & 0.84 & $(0.82,0.86)$ & I 219.5 & 188 & 29.0 & $(22.6,36.6)$ & 4199.5 & 493 \\
\hline $9-10$ & 0.82 & $(0.80,0.85)$ & I 881.0 & 232 & 38.3 & $(30.0,47.5)$ & 4908.9 & 588 \\
\hline II & 0.81 & $(0.78,0.84)$ & 939.9 & 127 & 46.6 & $(37.3,56.2)$ & 2015.2 & 283 \\
\hline$<5$ & 0.88 & $(0.86,0.90)$ & 982.9 & 179 & 12.0 & $(9.0,16.0)$ & 8165.1 & 1274 \\
\hline $5-11$ & 0.84 & $(0.82,0.87)$ & 4421.9 & 781 & 28.4 & $(22.2,35.5)$ & I5 562.7 & 2853 \\
\hline Total & 0.86 & $(0.83,0.88)$ & 5362.5 & 960 & 22.6 & $(I 7.5,28.6)$ & 23727.8 & 4127 \\
\hline
\end{tabular}

* According to the cut off value of International Zinc Nutrition Consultive Group ${ }^{20}$ 
Table III

Prevalence of Serum mineral deficiencies in Children aged I to I I Years by ARea of Residence and geographic region. National Health and Nutrition Survey 2006

\begin{tabular}{|c|c|c|c|c|c|c|c|}
\hline \multirow[b]{2}{*}{ Area } & \multirow[b]{2}{*}{ Region } & \multicolumn{4}{|c|}{ Prevalence of low iron stores, (ferritin $<12.0 \mu \mathrm{g} / \mathrm{L}$ ) } & \multicolumn{2}{|c|}{ Total population } \\
\hline & & $\mathrm{N}$ (thousands) & $n$ & $\%$ & $95 \% \mathrm{Cl}$ & $N$ (thousands) & $n$ \\
\hline Urban & & 2767.9 & 436 & 16.1 & $(12.8,20.0)$ & 17191.9 & 2767 \\
\hline Rural & & 1281.0 & 412 & 19.6 & $(15.9,24.0)$ & 6535.91 & 2149 \\
\hline & North & 1030.1 & 158 & 22.8 & $(18.1,28.3)$ & 4518.0 & 764 \\
\hline & Center & II 25.6 & 276 & 14.9 & $(12.1,18.3)$ & 7554.2 & |87| \\
\hline & Mexico City & 162.1 & II & 4.5 & $(2.3,8.6)$ & 3601.4 & 237 \\
\hline & South & 1594.7 & 403 & 19.8 & $(16.1,24.2)$ & 8054.2 & 2044 \\
\hline
\end{tabular}

\begin{tabular}{|c|c|c|c|c|c|c|c|}
\hline \multirow[b]{2}{*}{ Urban } & & \multicolumn{4}{|c|}{ Prevalence of tissue iron deficiency ( $s T f R>6 \mathrm{mg} / \mathrm{L}$ ) } & \multirow[b]{2}{*}{17191.9} & \multirow[b]{2}{*}{2767} \\
\hline & & 1702.0 & 282 & 9.9 & $(7.4,13.1)$ & & \\
\hline \multirow[t]{5}{*}{ Rural } & & 758.2 & 249 & 11.6 & $(8.9,15.2)$ & 6535.91 & $2|4|$ \\
\hline & Northern & 438.2 & 80 & 9.7 & $(6.7,13.9)$ & 4518.0 & 764 \\
\hline & Center & 914.1 & 228 & 12.1 & $(9.4,15.4)$ & 7554.2 & $|87|$ \\
\hline & Mexico City & 489.8 & 33 & 13.6 & $(9.4,19.4)$ & 3601.4 & 237 \\
\hline & Southern & 757.1 & 190 & 9.4 & $(7.1,12.3)$ & 8054.2 & 2036 \\
\hline
\end{tabular}

\begin{tabular}{|c|c|c|c|c|c|c|c|}
\hline \multirow[b]{2}{*}{ Urban } & & \multicolumn{4}{|c|}{ Prevalence of zinc deficiency* } & \multirow[b]{2}{*}{17191.9} & \multirow[b]{2}{*}{2341} \\
\hline & & 4760.9 & 648 & 27.7 & $(22.4,33.8)$ & & \\
\hline \multirow[t]{5}{*}{ Rural } & & 1650.1 & 478 & 25.2 & $(20.3,31.0)$ & 6535.9 & 1893 \\
\hline & Northern & 1462.0 & 237 & 32.4 & $(26.0,39.4)$ & 4518.0 & 733 \\
\hline & Center & 1958.6 & 463 & 25.9 & $(21.2,31.4)$ & 7554.2 & 1785 \\
\hline & Mexico City & 733.6 & 48 & 20.4 & $(14.0,28.2)$ & 3601.4 & 234 \\
\hline & Southern & 2095.0 & 385 & 26.0 & $(21.0,31.8)$ & 8054.2 & 1482 \\
\hline
\end{tabular}

\begin{tabular}{|c|c|c|c|c|c|c|c|}
\hline \multirow[b]{2}{*}{ Urban } & & \multicolumn{4}{|c|}{ Prevalence low serum copper concentrations $(<90 \mu \mathrm{g} / \mathrm{dL})$} & \multirow[b]{2}{*}{17191.9} & \multirow[b]{2}{*}{2269} \\
\hline & & 5197.7 & 684 & 30.2 & $(24.7,36.5)$ & & \\
\hline \multirow[t]{5}{*}{ Rural } & & 2036.4 & 587 & 31.2 & $(26.0,36.9)$ & 6535.91 & 1866 \\
\hline & Northern & 1584.7 & 259 & 35.1 & $(28.9,41.9)$ & 4518.0 & 732 \\
\hline & Center & 2220.1 & 402 & 29.4 & $(24.1,35.4)$ & 7554.2 & 1356 \\
\hline & Mexico City & I448.4 & 30 & 40.2 & $(28.9,53.0)$ & 3601.4 & 73 \\
\hline & Southern & 2361.8 & 580 & 29.3 & $(24.7,34.5)$ & 8054.2 & 1974 \\
\hline
\end{tabular}

\begin{tabular}{|c|c|c|c|c|c|c|c|}
\hline \multirow[b]{2}{*}{ Urban } & & \multicolumn{4}{|c|}{ Prevalence of low serum magnesium concentrations $(<0.75 \mathrm{mmol} / \mathrm{L})$} & \multirow[b]{2}{*}{17191.9} & \multirow[b]{2}{*}{2264} \\
\hline & & 4430.4 & 591 & 25.8 & $(20.1,32.3)$ & & \\
\hline \multirow[t]{5}{*}{ Rural } & & 1216.5 & 369 & 18.6 & $(14.2,24.0)$ & 6535.91 & 1863 \\
\hline & Northern & 1063.4 & 180 & 23.5 & $(18.1,30.0)$ & 4518.0 & 731 \\
\hline & Center & 1402.8 & 271 & 18.6 & $(14.1,23.9)$ & 7554.2 & $135 \mid$ \\
\hline & Mexico City & 1101.0 & 21 & 30.6 & $(19.2,44.6)$ & 3601.4 & 73 \\
\hline & Southern & 2017.7 & 488 & 25.1 & $(20.1,30.7)$ & 8054.2 & 1972 \\
\hline
\end{tabular}

* According to the cut off value of International Zinc Nutrition Consultive Group ${ }^{20}$ 
Table IV

LOGISTIC REGRESSION MODELS FOR MINERAL DEFICIENCIES AND POTENTIALLY PREDICTIVE VARIABLES in Children aged I to II years. National Health and Nutrition Survey 2006

Variables Odds Ratio p value $95 \% \mathrm{Cl}$

Dependent variable: Low iron stores, (ferritin $<12.0 \mu \mathrm{g} / \mathrm{L}$ ) $\quad N=4679$

Daily intakes

\begin{tabular}{|c|c|c|c|}
\hline Total iron (mg/d) & 0.98 & 0.32 & $(0.95,1.01)$ \\
\hline Phytates (mg/d) & 0.99 & 0.31 & $(0.99,1.00)$ \\
\hline Fiber (mg/d) & 0.99 & 0.85 & $(0.95,1.03)$ \\
\hline Vitamin C (mg/d) & 0.99 & 0.59 & $(0.99,1.00)$ \\
\hline Energy (kca/d) & 1.00 & 0.08 & $(0.99,1.00)$ \\
\hline C-reactive protein (mg/dL) & 0.88 & 0.00 & $(0.83,0.92)$ \\
\hline Age (years) & 0.83 & 0.00 & $(0.79,0.88)$ \\
\hline Gender* & 1.05 & 0.65 & $(0.83,1.32)$ \\
\hline \multicolumn{4}{|l|}{ Regions ${ }^{\ddagger}$} \\
\hline Center & 0.74 & 0.14 & $(0.50,1.10)$ \\
\hline Mexico City & 0.41 & 0.08 & $(0.15,1.12)$ \\
\hline Southern & 1.05 & 0.78 & $(0.7 \mathrm{I}, \mathrm{I} .54)$ \\
\hline Rural Area ${ }^{\S}$ & 0.78 & 0.17 & $(0.54, \mathrm{I} . \mathrm{II})$ \\
\hline \multicolumn{4}{|l|}{ Socioeconomic status } \\
\hline Middle & 0.93 & 0.60 & $(0.70,1.22)$ \\
\hline High & 0.63 & 0.03 & $(0.4 I, 0.97)$ \\
\hline Beneficiary of Liconsa program & 0.32 & 0.00 & $(0.17,0.61)$ \\
\hline $\begin{array}{l}\text { Beneficiary of Oportunidades } \\
\text { program }\end{array}$ & 1.08 & 0.90 & $(0.68, I .7 I)$ \\
\hline
\end{tabular}

Dependent variable:Tissue iron deficiency $(s T f R>6 m g / L)$

$N=4673$ deficiency $(s T f R<6 m g / d L)$

\begin{tabular}{|c|c|c|c|}
\hline \multicolumn{4}{|l|}{ Dietary intakes } \\
\hline $\operatorname{Iron}(\mathrm{mg} / \mathrm{d})$ & 1.00 & 0.80 & $(0.95,1.05)$ \\
\hline Phytates (mg/d) & 0.99 & 0.43 & $(0.99,1.00)$ \\
\hline Fiber $(\mathrm{mg} / \mathrm{d})$ & 0.98 & 0.68 & $(0.92,1.04)$ \\
\hline Vitamin C (mg/d) & 1.00 & 0.78 & $(0.99,1.00)$ \\
\hline Energy (Kcal/d) & 0.99 & 0.17 & $(0.99,1.00)$ \\
\hline Age (years) & 0.92 & 0.03 & $(0.85,0.99)$ \\
\hline Sex* & 1.36 & 0.08 & $(0.96,1.93)$ \\
\hline \multicolumn{4}{|l|}{ Regions $s^{\ddagger}$} \\
\hline Center & 1.04 & 0.85 & $(0.62,1.77)$ \\
\hline Mexico City & 1.96 & 0.03 & $(1.06,3.63)$ \\
\hline Southern & 0.68 & 0.18 & $(0.39,1.19)$ \\
\hline Rural Area ${ }^{\S}$ & 1.03 & 0.87 & $(0.71,1.49)$ \\
\hline \multicolumn{4}{|l|}{ Socioeconomic status $\#$} \\
\hline Middle $\ddagger$ & 0.79 & 0.26 & $(0.53,1.18)$ \\
\hline $\mathrm{High}^{\ddagger}$ & 0.91 & 0.77 & $(0.49,1.67)$ \\
\hline Beneficiary of Liconsa program & 0.80 & 0.48 & $(0.43, I .48)$ \\
\hline $\begin{array}{l}\text { Beneficiary of Oportunidades } \\
\text { program }\end{array}$ & 0.89 & 0.57 & $(0.6 \mathrm{I}, \mathrm{I} .3 \mathrm{I})$ \\
\hline
\end{tabular}

* Sex: I=boys; 0 =girls

$\ddagger$ Northern region is the comparison variable

$\S$ Urban area is the comparison variable

\# Score obtained by principal component analysis, according to household characteristics. Low socioeconomic status is the reference
Dependent variable: Low serum copper concentrations $\quad N=3807$ (<90 $\mu \mathrm{g} / \mathrm{dL})$

Dietary intakes

\begin{tabular}{llll} 
Copper $(\mathrm{mg} / \mathrm{d})$ & $\mathrm{I} .42$ & 0.34 & $(0.69,2.9 \mathrm{I})$ \\
\hline Fiber $(\mathrm{mg} / \mathrm{d})$ & $\mathrm{I} .0 \mathrm{I}$ & 0.55 & $(0.98, \mathrm{I} .03)$ \\
\hline Energy $(\mathrm{Kcal} / \mathrm{d})$ & $\mathrm{I} .00$ & 0.10 & $(\mathrm{I} .00, \mathrm{I} .00)$ \\
\hline Age $($ years $)$ & $\mathrm{I} .20$ & 0.17 & $(0.92, \mathrm{I} .55)$ \\
\hline Sex* & 0.76 & 0.01 & $(0.62,0.94)$ \\
C-reactive protein $(\mathrm{mg} / \mathrm{dL})$ & 0.89 & 0.00 & $(0.84,0.94)$
\end{tabular}

Regions ${ }^{\ddagger}$

$\begin{array}{llll}\text { Center } & 0.72 & 0.17 & (0.46, \mathrm{I} .14)\end{array}$

\begin{tabular}{llll} 
Mexico City & 1.43 & 0.29 & $(0.74,2.78)$ \\
\hline
\end{tabular}

$\begin{array}{llll}\text { Southern } & 0.64 & 0.04 & (0.41,0.99)\end{array}$

$\begin{array}{llll}\text { Rural Area }^{\S} & \text { I.0I } & 0.97 & (0.72, \mathrm{I} .40)\end{array}$

Socioeconomic status $\#$

$\begin{array}{llll}\text { Middle } & \text { I.09 } & 0.5 \mathrm{I} & (0.84, \mathrm{I} .43)\end{array}$

$\begin{array}{llll}\text { High } & 0.96 & 0.82 & (0.66,1.39)\end{array}$

$\begin{array}{lllll}\text { Beneficiary of Liconsa program } & 1.92 & 0.00 & (\mathrm{I} .24,2.97)\end{array}$

$\begin{array}{llll}\text { Beneficiary of Oportunidades } & \text { I.49 } & 0.04 & (\mathrm{I} .02,2.16)\end{array}$ program

Dependent variable: Low serum magnesium concentrations $\quad \mathrm{N}=380$ I (<0.75 mmol/L)

\begin{tabular}{|c|c|c|c|}
\hline \multicolumn{4}{|l|}{ Dietary intakes } \\
\hline Magnesium (mg/d) & 1.00 & 0.12 & $(0.99,1.00)$ \\
\hline Energy (kca/d) & 1.00 & 0.41 & $(0.99,1.00)$ \\
\hline Age (years) & 2.34 & 0.00 & $(1.75,3.12)$ \\
\hline Sex* & 0.90 & 0.38 & $(0.72, I .13)$ \\
\hline C-reactive protein $(\mathrm{mg} / \mathrm{dL})$ & 1.00 & 0.91 & $(0.98,1.02)$ \\
\hline \multicolumn{4}{|l|}{ Regions $\ddagger$} \\
\hline Center & 0.57 & 0.02 & $(0.35,0.93)$ \\
\hline Mexico City & 0.59 & 0.20 & $(0.26,1.33)$ \\
\hline Southern & 0.83 & 0.46 & $(0.50,1.36)$ \\
\hline Rural Area ${ }^{\S}$ & 0.97 & 0.88 & $(0.66 \quad I .42)$ \\
\hline \multicolumn{4}{|l|}{ Socioeconomic status ${ }^{\#}$} \\
\hline Middle & I.II & 0.48 & $(0.83,1.50)$ \\
\hline High & 1.04 & 0.86 & $(0.69, \mathrm{I} .55)$ \\
\hline Beneficiary of Liconsa program & 0.79 & 0.37 & $(0.47,1.33)$ \\
\hline $\begin{array}{l}\text { Beneficiary of Oportunidades } \\
\text { program }\end{array}$ & 0.80 & 0.22 & $(0.561 .14)$ \\
\hline
\end{tabular}

program 
The prevalence of TID as assessed by sTfR (11\%) differed from LIS (15.7\%) as indicated by ferritin concentrations because the two indicators measure the iron status in different body compartments: ferritin reflects the hepatic stores ${ }^{25}$, while sTfR is an indicator of iron available at tissue level. ${ }^{26}$ Several indicators of iron nutritional status, ferritin among them, are altered by inflammation, ${ }^{27}$ therefore, prevalences should be adjusted for inflammation indicators such as CRP. Adjustment was carried out in this analysis and had a marginal impact on the prevalence of iron deficiency.

The protective role of being beneficiary of Liconsa program for iron deficiency, suggests the effectiveness of this program in preventing and controlling iron deficiency. Liconsa is a social program aimed at improving the micronutrient status (iron, zinc, and folic acid) through fortified milk. It is targeted to low-income population who are nutritional vulnerable such as children $<12$ years old, pregnant or teenager mothers who are breastfeeding their babies, elderly and handicap individuals. Nationwide, Liconsa assists over 4 million children. The protective effect of Liconsa on iron status is supported by previous randomized trials on the efficacy and effectiveness of this program to decrease iron deficiency in children. ${ }^{28,29}$

On the other hand, the Oportunidades program did not show an impact on anemia. The objective of Oportunidades is to avoid the transmission of poverty in families that live in extreme poverty conditions through incentives for investments in human capital formation. Support includes a conditional monetary transfer. The conditions to support families include attendance to basic health services and nutritional surveillance. In addition, the program supplies the families of young children or pregnant women with a fortified (iron, zinc, C vitamin, and folic acid) food supplement.

Our result are contrary to effects of the Oportunidades program on anemia reduction documented in rural areas in a randomized controlled effectiveness study ${ }^{30}$ but is in line with an effectiveness evaluation in urban areas ${ }^{31}$ and with a 2008 report which shows high prevalence of anemia in rural children younger than 2 years $(27-40 \%)$ receiving the benefits of this program. ${ }^{32}$ However, since this is a cross-sectional analysis and the beneficiaries of Oportunidades are the worst-off population, we cannot exclude residual confounding, even when adjusting for indicators of living conditions.

\section{Nutritional status of zinc}

A high prevalence of zinc deficiency in this age group ( $\geq 20 \%$ to be a risk of zinc deficiency) was found, ${ }^{7}$ making mandatory a public intervention. ${ }^{20}$ Several public interventions with zinc were initiated in México since 1999. The efficacy of three nutritional supplements with a similar content of multiple micronutrients, including zinc, were tested in a clinical trial in urban Mexican preschoolers. A micronutrients syrup and "sprinkles" improved serum zinc concentrations after 4 mo of treatment $(p<0.05)$.The fortified food Nutrisano had a non significant positive trend $(p>0.05)$, but was effective at improving serum zinc in zinc deficient children. ${ }^{33}$ The programs Liconsa and Oportunidades serve several hundred thousand of children with zinc fortified food. In spite of that, results from the 1999 show that prevalence then was $25.3 \%$ in children 6 months to 11 years, ${ }^{14}$ very similar to the prevalence found in 2006 for the same age group $(26.6 \%)$, indicating that the prevalence did not decrease between 1999 and 2006. The persistence of zinc deficiency, despite these public efforts, calls for re-examining the ongoing strategies to tackle zinc deficiency. The high prevalence of zinc deficiency may be associated with the high dietary intake of inhibitors of zinc absorption, such as phytates, calcium, and fiber in the Mexican diet. ${ }^{34,35}$ There is a need for studies aimed at identifying actions to improve the effectiveness of the micronutrient-fortified foods provided by governmental programs or to test alternative strategies.

\section{Nutritional status of copper and magnesium}

The proportion of copper and magnesium LSC were also high in this sample; nonetheless, we found few population-based publications for comparisons. A study in Californian children 1-3 years old, reported a prevalence of copper LSC which was very small (below 1\%), using the same cut-off point employed in our study. ${ }^{36}$ A prevalence of $4.45 \%$ was reported in Venezuelan children younger than 15 years; however, the cut-off point used in the Venezuelan study was lower $(<87 \mu \mathrm{g} /$ dL) than our study $(90 \mu \mathrm{g} / \mathrm{dL})$, and is therefore non comparable $^{37}$. The prevalence reported herein is higher than in those reports. Our determinations of serum copper were calibrated against an internationally certified standard (NIST 3131a) and therefore are reliable. Such a high prevalence is in line with the high iron and zinc deficiencies reported herein, since dietary sources for all three minerals are similar. The relevance of the nutritional status of copper stems from its essential role in the cellular respiration process and in the development and function of the nervous system. ${ }^{12}$

An additional issue is that children beneficiaries of Liconsa and Oportunidades had a significant risk for copper LSC. None of the foods distributed by those pro- 
grams are fortified with copper and are far from fulfilling the copper DRI $(340 \mu \mathrm{g} / \mathrm{d}) .{ }^{38}$ The negative association between copper LSC and consumption of Liconsa milk may be explained by the known interaction between copper and iron metabolism. Increased iron absorption decreases circulating levels of copper, because of the role of copper in the transport and absorption of iron.

Serum copper levels tend to decrease with increasing body's use of iron from fortified milk, which improves anemia as demonstrated by this and other studies.

This is the first time that data on magnesium LSC is reported in Mexican population. The prevalence resulted high (one quarter of children 1-11 years of age). A study in Vietnamese rural children aged 6-8 years showed a prevalence of magnesium LSC of 59.5\%, higher than in this sample.

Another study conducted in Iranian individuals from 3 to 19 years found a magnesium LSC prevalence of $5.9 \%$ and $5.6 \%$ in males and females respectively. ${ }^{21}$

A study in India about lead exposition effects found a magnesium LSC prevalence of $41.7 \%$ in those children who suffer subclinical lead intoxication. ${ }^{40}$

Despite our results indicating high proportions of children with cooper and magnesium LSC, the functional and health significance of these serum values found in our population needs further examination.

In summary, a high percentage of the population younger than 12 years suffers from iron and zinc deficiency, and had low serum concentrations of copper and magnesium. Iron and zinc deficiencies are of great concern for public health in Mexico. Zinc deficiency is particularly worrisome because its prevalence has not diminished between 1999 and 2006, despite the large distribution of food fortified with zinc by government food assistance programs to targeted population. Fortified foods have proved effective in decreasing anemia. For the first time, information about the prevalence of copper and magnesium LSC in a representative sample of Mexican children is reported. The high proportion of children with magnesium LSC call for further research into their consequences on health, to assess whether there is a need for a public health intervention. Altogether, these results offer relevant information that can be of interest for decision makers.

\section{Acknowledgments}

We are grateful to Ignacio Mendez Gómez Humarán for his statistical advice.

Declaration of conflict of interests. The authors declare that they have no conflict of interests.

\section{References}

I. Investing in the future. A united call to action on vitamin and mineral deficiencies. Global report 2009. Ottawa, Canada: Micronutrient Initiative, 2009.

2. Black RE, Allen L H, Bhutta Zq A, Caulfield LE, de Onis M, Ezzati M, et al. Maternal and child undernutrition: global and regional exposures and health consequences. Lancet 2008; 37I(9608):243-260.

3. Allen L, Benoist B, Dary O, Hurrel R. Guidelines on food fortification with micronutrients. Switzerland: World Health Organization, Food and Agricultural Organization of the United Nations, 2006.

4 Stoltzfus RJ. Iron deficiency: global prevalence and consequences. Food Nutr Bull 2003;24:S99-I03.

5. Scholl TO, Reilly T. Anemia, iron and pregnancy outcome. J Nutr 2000; 130:443S-447S.

6. Brown KH, Peerson JM, Baker SJ, Hess SY. Preventive zinc supplementation among infants, preschoolers, and older prepubertal children. Food Nutr Bull 2009; 30:S12-40.

7. Hess SY, Lönnerdal B, Hotz C, Rivera JA, Brown K. Recent advances in knowledge of zinc nutrition and human health. Food Nutr Bull 2009; 30:S5-II.

8. Swaminathan R. Magnesium Metabolism and its disorders. Clin Biochem Rev 2003;24: 47-66

9. Arnaud JM. Update on the assessment of magnesium status. B J Nutr 2008;99 Suppl 3:S24-36.

I0. Huerta GM, Roemmich NJ, Kington LM, Bovbjerg EV, Weltman LA, Holmes FV, et al. Magnesium deficiency Is associated with insulin resistance in obese children. Diabetes Care 2005;28: I I75-I I8I.

II. Fraga C. Relevance, essentiality and toxicity of trace elements in human health. Mol Aspects Med 2005;26:235-244.

I2. Desai V, Kaler SG. Role of copper in human neurological disorders. Am J Clin Nutr 2008;88:855S-8S.

13. Cater MA, Mercer JFB. Copper in mammals: mechanisms of homeostasis and pathophysiology. Molecular Biology of Metal Homeostasis and Detoxification. In: Tamás MJ, Martinoia E (ed). Topics in Current Genetics. Heidelberg, Germany: Springer-Verlag Heiderlberg, 2005: I0I-129. 14. Villalpando S, García-Guerra A, Ramírez-Silva Cl, Mejía-Rodríguez F, Matute G, Shamah-Levy T, et al. Iron, zinc and iodide status in Mexican children under 12 years and women 12-49 years of age. A probabilistic national survey. Salud Publica Mex 2003;45 suppl 4:520-529.

I5. Palma O, Shamah-Levy T, Franco A, Olaiz-Fernández G, Méndez I. Metodología. In: Encuesta Nacional de Salud y Nutrición (ENSANUT-2006).

Cuernavaca, México: Instituto Nacional de Salud Pública, 2006:19-33.

16. Resano E, Méndez I, Shamah T, Rivera J, Sepúlveda J. Methods of the National Nutrition Survey 1999. Salud Publica Mex 2003:45 suppl 4:558-564.

17. Rodríguez-Ramírez S, Mundo-Rosas V, Jiménez-Aguilar A, ShamahLevy T. Methodology for the analysis of dietary data from the Mexican National Health and Nutrition Survey 2006. Salud Publica Mex 2009;5I suppl 4:523-529.

18. World Health Organization. Iron deficiency anaemia: assessment, prevention, and control. A guide for programme managers. Geneva: WHO, 200I. WHO/NHD/0I.3.

19. Beguin Y. Soluble transferrin receptor for the evaluation of erythropoiesis and iron status. Clin Chim Acta 2003;329:9-22.

20. International Zinc Nutrition Consultative Group. Assessment of the risk of zinc deficiency in populations and options for its control. Technical Document \#I. Food Nutr Bull 2004;25:S94-S203.

2I. Ghasemi A, Syedmoradi L, Zahediasl S, Azizi F. Pediatric reference values for serum magnesium levels in Iranian subjects. Scand J Clin Lab Invest 2010;70:415-420. 
22. Cordano A. Clinical manifestations of nutritional copper deficiency in infants and children. Am J Clin Nutr 1998;67:SI0I2-SI0I6.

23. Organización Panamericana de la Salud. Principios de orientación para la alimentación complementaria del niño amamantado. Unidad de nutrición, salud de la familia y comunidad. Washington DC: OPS, 2003. 24. Neufeld LM, Ramakrishnan U. Specific Strategies to Address Micronutrient Deficiencies in the Young Child: Targeted Fortification. In: Pettifor JM, Zlotkin S eds. Micronutrient Deficiencies during the Weaning Period and the First Years of Life. Nestlé Nutrition Workshop Series, Pediatric Program. Vevey: Nestec Ltd and Basel: S Karger 2004;54:83-103. 25. Lee EJ, Oh EJ, Park YJ, Lee HK, Kim BK. Soluble transferrin receptor (sTfR), Ferritin, and sTfR/log ferritin index in anemic patients with nonhematologic malignancy and chronic Inflammation. Clin Chem 2002;48: I| |8-| | $2 \mid$

26. Skikne BS, Flowers $\mathrm{CH}$, Cook JD. Serum transferrin receptor: a quantitative measure of tissue iron deficiency. Blood 1990;75:1870-1876. 27. Gibson RS. Assessment of Iron Status. Gibson RS, ed. Principles of Nutritional Assessment. New York - Oxford: Oxford University Press 2005:443-476.

28. Villalpando S, Shamah T, Rivera JA, Lara Y, Monterrubio E. Fortifying milk with ferrous gluconate and zinc oxide in a public nutrition program reduced the prevalence of anemia in toddlers. J Nutr 2006;136:2633-2637. 29. Rivera JA, Shamah T, Villalpando S, Monterrubio E. Effectiveness of a large-scale iron-fortified milk distribution program on anemia and iron deficiency in low-income young children in Mexico. Am J Clin Nutr 2010;91:431-439.

30. Rivera JA, Sotres-Alvarez D, Habicht JP, Shamah T, Villalpando S Impact of the Mexican Program for Education, Health and Nutrition (Progresa) on rates of growth and anemia in infants and young children. A randomized effectiveness study. JAMA 2004;291:2563-2570.

31. Leroy J, García-Guerra A, Garcia R, Dominguez C, Rivera JA, Neufeld LM. The Oportunidades Program Increases the Linear Growth of Children Enrolled at Young Ages in Urban Mexico. J Nutr 2008; 138:793-798.
32. Neufeld LM, Mejia F, Fernández Gaxiola AC, García Guerra A, Méndez I, Domínguez CP. Diagnóstico situacional del estado nutricio de niños menores de dos años de edad y de sus madres, beneficiarios de Oportunidades en zonas rurales. En: Evaluación externa del Programa Oportunidades 2008. A diez años de intervención en zonas rurales (1997-2007). Vol 2. El reto de la calidad de los servicios: resultados en salud y nutrición. Cuernavaca: Instituto Nacional de Salud Pública, 2008. 33. García-Guerra A, Rivera-Dommarco J, Neufeld L, Domínguez-Islas CP. Effect of three supplements with equal micronutrient content on serum zinc concentrations in Mexican children. FASEB J 2009;23:917.13. 34. Krebs N. Overview of zinc absorption and excretion in the human gastrointestinal tract. J Nutr 2000;।30:SI374-SI377.

35. World Health Organization. Zinc. Vitamin and mineral requirements in human nutrition. Geneva: World Health Organization, 2004:230-245. 36. Schneider JM, Fujii ML, Lamp CL, Lönnerdal B, Zidenberg-Cherr S. The prevalence of low serum zinc and copper levels and dietary habits associated with serum zinc and copper in 12- to 36-month-old children from low-income families at risk for iron deficiency. J Am Diet Assoc 2007;107:1924-1929.

37. Rodríguez D, Papale J, Dellan G, Torres M, Berné Y, Mendoza N, et al. Deficiencia de zinc y cobre en menores de 15 años en una población rural de Venezuela. Boletín Médico 2004:20:55-60.

38. Rosado J. Ingestión dietética recomendada de cobre. En: Bourgues $\mathrm{H}$, Casanueva E, Rosado J, ed. Ingestión de nutrimentos para la población mexicana, bases fisiológicas. México D.F.: Médica Panamericana, 2005:273-280.

39. Nhien NV, Khan NC, Yabutani T, Ninh NX, Chung le TK, Motonaka J, et al. Relationship of low serum selenium to anemia among primary school children living in rural Vietnam. J Nutr Sci Vitaminol 2008;54:454-459. 40. Srinivasa-Reddy Y, Pullakhandam R, Radha-Krishna KV, Uday-Kumar $P$, Dinesh-Kumar B. Lead and essential trace element levels in school children: a cross-sectional study. Ann Hum Biol 201 I;38:372-377. 